\title{
CARACTERIZACIÓN DE UN NANOCOMPUESTO DE CAUCHO NATURAL REFORZADO CON ARCILLA
}

\section{CHARACTERIZATION OF NATURAL RUBBER NANOCOMPOSITE REINFORCED BY CLAY}

\author{
Chirinos Collantes Hugo David ${ }^{1}$
}

RESUMEN

El presente trabajo caracteriza un nanocompuesto obtenido a partir de látex de caucho natural reforzado con arcilla. Se estudiaron las propiedades de sorción, mecánicas y térmicas. En la sorción se observó que cuanto mayor es la cantidad de arcilla, se absorbe menos cantidad de solvente. Las propiedades mecánicas mostraron un material muy tenaz con alto porcentaje de arcilla y baja elasticidad. Los ensayos termogravimétricos mostraron que la pérdida de masa de los nanocompuestos es menor con el aumento de la concentración de arcilla.

Palabras clave.- Nanocompuesto, Caucho natural, Arcilla, Caracterización

\section{ABSTRACT}

This contribution shows the caracterization of nanocomposites from natural rubber latex reforcing by clay particles. The sortion, the mechanical and the thermical proporties were studied. In the sortion analysis, when increase the concentration clay the nanomaterial absorved low quantity of solvent. The mechanical properties analysis show a high hardness material when increase the clay but with low elasticity. The thermogravimetrical analysis shows low mass sold of nanomaterial by increasing clay concentration.

Key words.- Nanocomposite, Natural rubber, Clay, Characterizacion.

\section{INTRODUCCIÓN}

Nanocompuestos son todos los sistemas que presentan interfaces en escala nanométrica [1;6]. Hay un gran interés tecnológico por los nanocompuestos polímeros-orgánicos/cargasinorgánicas, más específicamente en los sistemas polímeros-arcillas $[10 ; 11 ; 13]$. Los trabajos pioneros, de $\mathrm{Wu}$ [14] y de Lan [9] describen la síntesis de nanocompuestos convencionales, ellos realizaron estudios en varios sistemas poliméricos adicionando nanotubos, nanofibras o nanopartículas de $\mathrm{SiO}_{2}$ nanométrico [2]. El gran interés por los sistemas con arcillas se debe a su estructura laminar con dimensión nanométrica $[1,6,10,11]$. El resultado de la relación entre el área superficial y el espesor es una gran área interfacial que permite un alto grado de interacción con el polímero, lo que implica un alto índice de refuerzo para poca carga incorporada. La mayor parte de los estudios se realizan utilizándose arcillas sintéticas del tipo Motmorillonita-sódica pero, las motmorillonitas naturales, comúnmente identificadas como bentonitas, son las más importantes $[5 ; 12]$. La capacidad de intercambio de cationes de las arcillas permite la rápida formación de un coloide con la adición de agua. Es necesario modificar las arcillas para que presenten superficies organofílicas, ya que

\footnotetext{
${ }^{1}$ Docente de la Facultad de Ingeniería Ambiental de la Universidad Nacional de Ingeniería.
} 
la mayoría son hidrofóbicas, con la finalidad de compatibilizar la carga y el polímero a ser reforzado $[4 ; 8]$. Así, dependiendo de la compatibilidad entre el polímero y la arcilla, se obtienen dos tipos de materiales: membranas nanoestructuradas intercaladas y/o exfoliadas. Hay tres métodos de obtención: intercalación del polímero por fusión y acción mecánica en extrusora [6]; polimerización "in situ" [3] utilizados en sistemas polimerizados por presión (autoclave) y obteniendo estructuras intercaladas; intercalación por solución [7] según la solubilidad del polímero en el solvente compatible con la superficie modificada de la arcilla o no modificada.

La incorporación de arcillas en polímeros a base de agua se estudió por Valadares y colaboradores [12] en mezclas de dispersiones acuosas de arcillas y látex. El látex de caucho natural está constituido por una dispersión coloidal del polímero cis 1,4 poliisopreno en medio acuoso. La partícula de látex, de tamaño nanométrico, contiene al menos una terminación hidrofílica y otra hidrofóbica. De esta manera, la compatibilidad entre el polímero y la carga en el estado coloidal ocurre naturalmente, permitiendo un excelente grado de homogenización [5].

Los sistemas poliméricos a base de agua también permiten la obtención de caucho sólido a través de la desestabilización química de la dispersión coloidal, las partículas del polímero se aglomeran formando bloques de material denso. Los estudios de $\mathrm{Wu}$ [15] utilizan únicamente la etapa de la coagulación para la obtención de nanocompuestos.

El siguiente estudio reporta la caracterización de un nanocompuesto obtenido por el proceso de intercalación por solución sin compatibilizar la carga.

\section{MATERIALES Y METODOS}

La metodología para la preparación del nanocompuesto de caucho natural y arcilla está constituido de tres etapas principales: la dispersión de la arcilla en agua, la homogenización del látex con la dispersión de arcilla exfoliada y el secado de la mezcla.

\section{Materias primas}

El látex caucho natural utilizado fue de grado comercial, concentrado a $60 \%$, del tipo alta concentración de amoniaco (HA, $0.7 \%$ ), proveniente de las seringueras del Planalto Central del Brasil. La arcilla montmorillonita- $\mathrm{Na}^{+}, \quad$ conocida comercialmente como bentonita sódica fue de grado comercial. Se controla la calidad de la arcilla retirando una muestra para realizar análisis de entumecimiento (hinchamiento) con agua desionizada.

\section{Método de preparación del nanocompuesto}

La metodología de preparación consiste en preparar la suspensión de arcilla en agua desionizada en concentración de $1 \%$ hasta $5 \%$ del producto seco. Se usó un dispersor de tipo cowles (2500rpm) controlando el tiempo de agitación para conseguir el hinchamiento total de la partículas de arcilla. En seguida, se filtró la suspensión en filtros de tipo bag de 50 micrones. Se controló el pH de la suspensión de arcilla manteniéndolo en 10, para luego adicionarlo al látex de caucho natural.

En esta etapa se estudian proporciones de arcilla y látex de caucho natural en un intervalo de $10-20 \%$ de concentración de sólidos totales y de $2-5 \%$ de caucho seco del nanocompuesto producido. Por último, se prepara el nanocompuesto en agitador de baja rotación $(100 \mathrm{rpm})$ de tipo ancla y con alto torque. Es importante monitorear la estabilidad del nanocompuesto producido para evitar la coagulación espontánea del mismo.

\section{Preparación de las membranas del nanocompuesto obtenido}

Las membranas de caucho obtenidas a partir del nanocompuesto con $2 \mathrm{~mm}$ de grosor son confeccionadas por el método casting. Donde la suspensión del nanocompuesto se derrama sobre placas de vidrio (120x120x40mm), limpias, secas y en superficies niveladas. La coacervación, que consiste en la unión de las partículas de caucho y arcilla dando origen al sólido, ocurre a temperatura ambiente y durante $48 \mathrm{~h}$, dependiendo de los siguientes factores: grosor; temperatura y la 
humedad del medio ambiente. Luego se retira la membrana de la placa de vidrio y se realizan las etapas de lixiviación húmeda y seca. Luego se cortan en cuerpos de prueba para realizar los ensayos mecánicos.

\section{RESULTADOS Y DISCUSIÓN}

La Figura 1 presenta las curvas de sorción de xileno de las muestras analizadas. Los nanocompuestos con mayor proporción en arcilla absorben menos solvente de los que tienen menor cantidad. Además se observa que el caucho puro absorbe mayor cantidad de solvente.

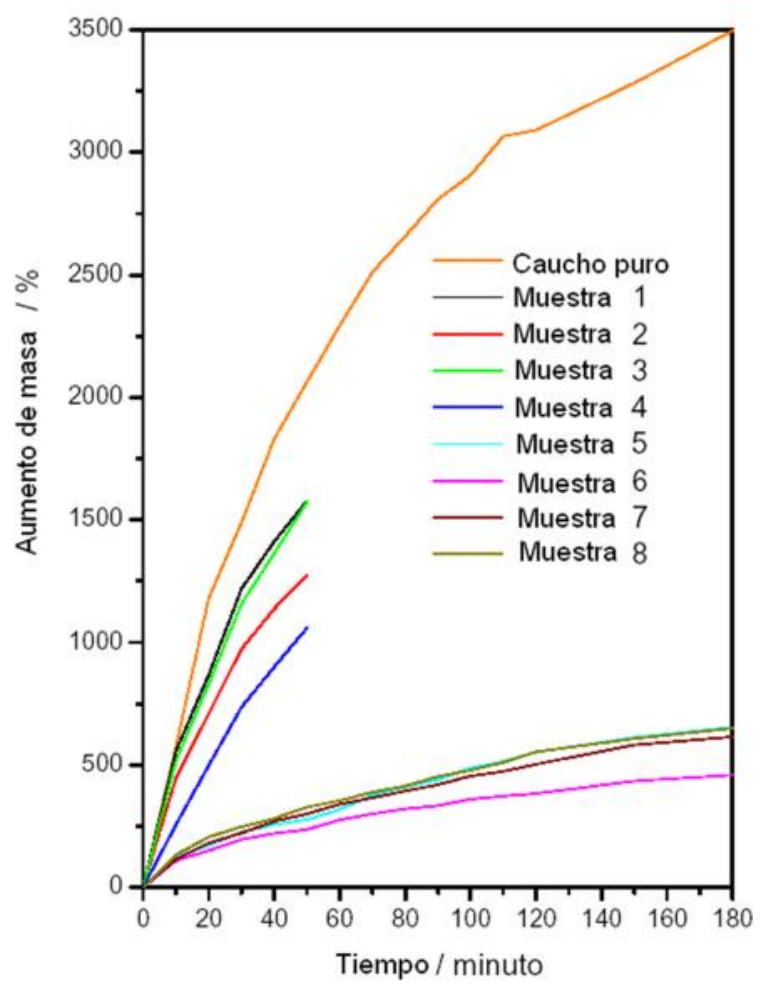

Fig.1 Sorción de xileno en los nanocompuestos y en el caucho natural.

Se observó que la concentración de arcilla afecta en el entumecimiento de las muestras. La Figura 2 muestra el estiramiento sufrido por las muestras en las tres direcciones durante la sorción de xileno.

Las direcciones $\mathrm{x}$ e y forman el plano de secado de los materiales y la dirección z es normal a ese plano.
Las curvas de caucho puro se cruzan, estando prácticamente sobrepuestas unas con las otras, indicando que el caucho puro se hincha simultáneamente en todas las direcciones al mismo tiempo, o sea, es isotrópica en el entumecimiento.

En la muestra 1, 5phr (parte por ciento de resina) de arcilla el estiramiento en $\mathrm{z}$ es superior al estiramiento en $\mathrm{x} y$ en $\mathrm{y}$, indicando que la muestra hincha preferentemente en $\mathrm{z}$.

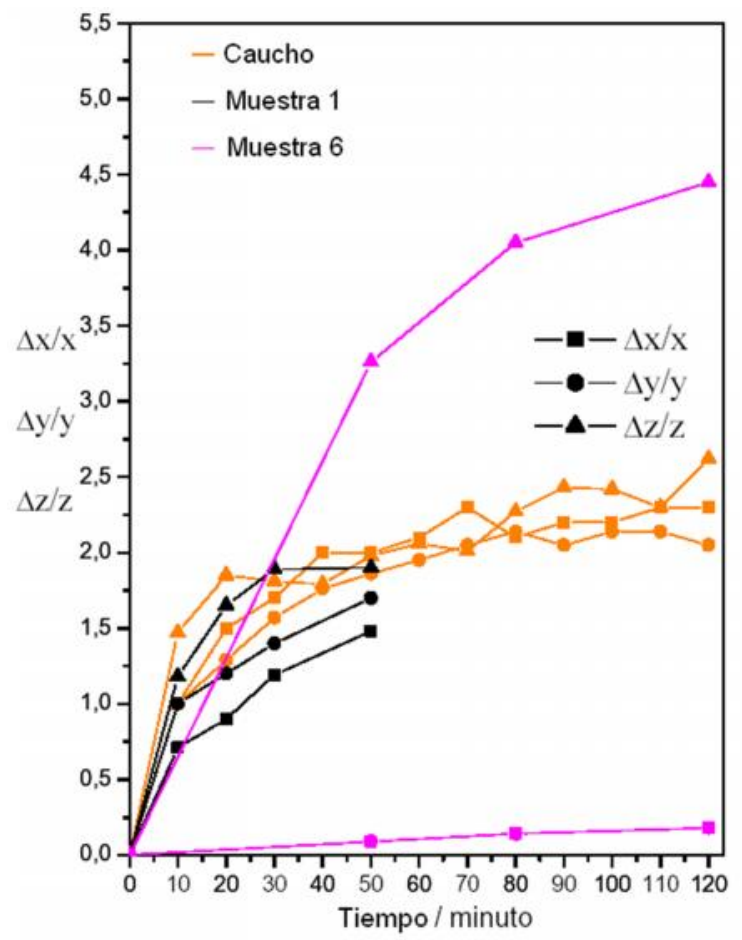

Fig. 2 Estiramiento en las direcciones $x, y, z$ durante la sorción de xileno vs. tiempo.

La muestra 6 (30phr de arcilla) presenta claramente una anisotropía de entumecimiento debido a que posee un estiramiento en $\mathrm{z}$ mucho mayor que en $\mathrm{x} o$ en y a medida que absorbe el xileno, lo que demuestra que el nanocompuesto se hincha preferentemente en la dirección perpendicular al plano de secado de la película.

La resistencia a la sorción de xileno es debido a que las láminas de arcilla actúan como una barrera en la penetración del solvente, posibilitando que la difusión del solvente ocurra la estructura laminar 
arcilla. La Figura 3 muestra las curvas de tracción, donde los comportamientos son muy distintos entre el caucho natural, las muestras con $5 \mathrm{phr}$ de arcilla y las muestras con $30 \mathrm{phr}$.

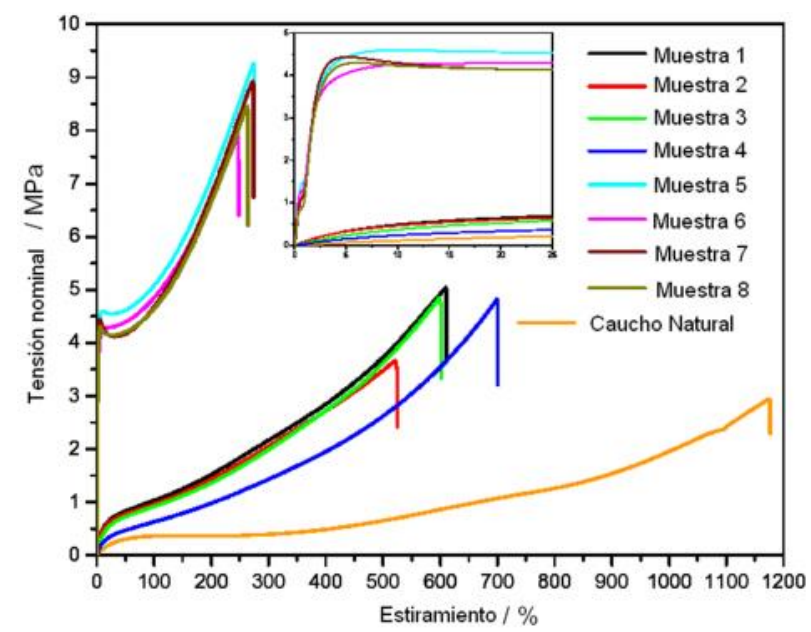

Fig. 3 Curvas de tensión-deformación del caucho natural y de los nanocompuestos.
Los valores promedios del modulo de Young, estiramiento máximo y tensión en la ruptura obtenidos de las curvas se presentan en la Tabla 1.

Tabla 1. Propiedades mecánicas del caucho puro y de los nanocompuesto.

\begin{tabular}{llll}
\hline Muestra & Módulo de Young, MPa & Estiramiento máximo, \% & $\begin{array}{l}\text { Tensión en la ruptura, } \\
\text { MPa }\end{array}$ \\
\hline Caucho & $0.9 \pm 0.1$ & $1132 \pm 46$ & $2.8 \pm 0.3$ \\
$1(5 \mathrm{phr})$ & $6.5 \pm 0.7$ & $599 \pm 21$ & $4.9 \pm 0.3$ \\
$2(5 \mathrm{phr})$ & $6.5 \pm 0.6$ & $496 \pm 18$ & $3.4 \pm 0.2$ \\
$3(5 \mathrm{phr})$ & $4.6 \pm 0.2$ & $608 \pm 14$ & $5.0 \pm 0.2$ \\
$4(5 \mathrm{phr})$ & $1.9 \pm 0.6$ & $645 \pm 30$ & $4.0 \pm 0.5$ \\
$5(30 \mathrm{phr})$ & $243 \pm 34$ & $243 \pm 28$ & $8.0 \pm 1.1$ \\
$6(30 \mathrm{phr})$ & $223 \pm 71$ & $245 \pm 28$ & $7.9 \pm 1.1$ \\
$7(30 \mathrm{phr})$ & $228 \pm 47$ & $243 \pm 35$ & $8.1 \pm 1.1$ \\
$8(30 \mathrm{phr})$ & $203 \pm 49$ & $251 \pm 93$ & $7.9 \pm 0.3$ \\
\hline
\end{tabular}

La curva de tracción del caucho natural muestra que la tensión aumenta hasta un estiramiento del $75 \%$; entonces, la tensión permanece constante hasta la deformación del 300\%. Encima de ese valor la tensión aumenta hasta la ruptura del material, que ocurre a 2.8MPa. El caucho natural presenta un bajo modulo de Young y alta deformación, debido que no está vulcanizado. Los nanocompuestos con $5 \mathrm{phr}$ de arcilla presentaron un alto modulo (de 2 a 8 veces) con respecto al caucho puro. La ruptura ocurre en estiramientos de cerca de 500\%-600\%. Estos nanocompuestos no presentan la región donde la tensión permanece constante con el estiramiento. Los nanocompuestos con 30phr de arcilla presentaron un alto modulo inicial, un valor cercano de 250 veces mayor que el caucho puro. En el inicio del estiramiento, hay un gran aumento de tensión hasta que el material llegue al punto de fluir (en el estiramiento de $7.5 \%$ sobre una tensión de $4.6 \mathrm{MPa}$ ), ocurriendo después una leve disminución en la tensión. El material se estira hasta la ruptura con una tensión de 2.5 veces al caucho puro con un 
estiramiento 5 veces menor al caucho puro. Pero el grado de estiramiento es bajo, cerca de $245 \%$, mostrando que el material posee alta tenacidad.

Con la finalidad de estudiar las propiedades térmicas de los nanocompuestos y compararlos con el caucho natural se realizaron ensayos termogravimétricos de la arcilla, de las muestras 1, 4, 6, 7 y del caucho puro. Los termogramas se muestran en la Figura 4.

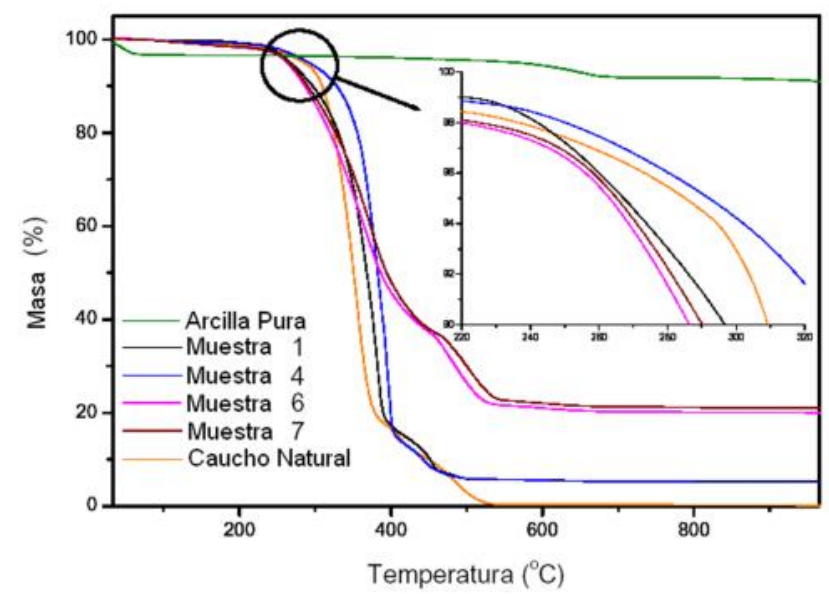

Fig. 4 Curvas termogravimétricas indicando el inicio de la degradación.

La curva termogravimétrica de la montmorillonita pura presenta una pérdida de masa hasta la temperatura de $100^{\circ} \mathrm{C}$, debido a la evaporación del agua. Otra pérdida de $5 \%$ en masa entre las temperaturas de $280^{\circ} \mathrm{C}$ y $680^{\circ} \mathrm{C}$ debido al agua molecular de los iones interlaminares, siendo más pronunciada en la región entre $550^{\circ} \mathrm{C}$ y $680^{\circ} \mathrm{C}$. Las curvas termogravimétricas del caucho y de los nanocompuestos presentan perdida a la temperatura de $285^{\circ} \mathrm{C}$ debido a que los materiales están secos. Pero, presentan dos zonas de pérdidas, la primera zona, entre las temperaturas de $285^{\circ} \mathrm{C}$ y $555^{\circ} \mathrm{C}$, debido a la pirólisis oxidativa del caucho y otra zona entre $475^{\circ} \mathrm{C}$ y $555^{\circ} \mathrm{C}$, debido a la descomposición final. Encima de esa temperatura no se observa pérdida de masa significativa, restando apenas la materia inorgánica. Los nanocompuestos de $5 \mathrm{phr}$ comienzan a perder masa a temperaturas mayores que la del caucho; mientras que en los nanocompuestos de 30phr, de modo antagónico, comienzan a perder masa a partir de temperaturas más bajas que del caucho. Esto puede ser debido a la presencia de agua molecular de los iones en las interfaces del nanocompuesto o a la descomposición catalítica del caucho.

\section{CONCLUSIONES}

Se pueden preparar Nanocompuestos de caucho natural reforzado con montmorillonita sódica. Los nanocompuestos obtenidos presentan diferentes estructuras (intercalada y/o exfoliada ordenada) dependiendo de la concentración de arcilla. Poseen gran versatilidad de propiedades mecánicas en función de la cantidad de arcilla. Los nanocompuestos poseen mayor resistencia a solventes orgánicos que el caucho puro. Las Láminas están orientadas en las placas actuando de esta manera como una barrera a solventes. Se puede suponer que actúen de la misma forma en relación a la permeabilidad de gases.

\section{REFERENCIAS}

1. Alexandre, M.; Dubois, P., "Polymer-layered Silicate Nanocomposites: Preparation, Properties and Uses of a New Class of Materials" Mat. Sci. Eng. 1(28) 2000.

2. Amalvy, J. J; Percy, M. J.; Arnes, S. P; Leite, A. P.; Galembeck, F., "Characterization of the nanomorphology of Polymer-silica colloidal nanocomposites using electron spectroscopy imaging" Langmuir 1175 (21) 2005.

3. Dolozier, D. M.; Orwoll, R. A.; Cahoon, I. F.; Jhonston , N. L.; Amithm, J. G.; Connell, J. W., "Polymer, 43", pp. 813, 2002.

4. Elpass, C. W.; Peiffer, D. G.; Kresge, E. N.; Hsieh, D. T.; Chludzinski, J. J.; Liang, K. S., "Nanocomposite Materials" US Pat. 5.883.173; 1999.

5. Galembeck, F.; Valadares, L. F.; Rippel, M. M.; Murakami, M. M., "Produção de nanocompósitos de Termoplásticos ou Elastômeros com argilas intercaladas ou esfoliadas, a partir de látices". INPI, PI 301193; 2003.

6. Giannelis, E., "Polymer Layered Silicate Nanocomposites" Adv. Mater. 29 (8) 1996. 
7. Jeon, H. S.; Rameshwaram, J. K.; Kim, G.; Weinkauf, D. H., "Polymer". 44, 5749. 2003.

8. Kamigaito, O.; Fukushia, Y.; Doi, H., "Composite Material Composed of Clay Mineral and Organic High Polymer and Method for Producing the Same" US Pat. 4.472.538; 1984.

9. Lan, T.; Pinnavaia, T. J., "Clay-reinforced Epoxy Nanocomposites" Chem. Mater. 2216 (6) 1994.

10. LeBaron, P. C.; Wang, Z.; Pinnavaia, T. J., "Polymer-layered Silicate Nanocomposites: an Overview". Appl. Clay Sci. 11 (15) 1999.

11. Ray, S. S.; Okamoto, M., "Polymer/layered Silicate Nanocomposites: a Review from Preparation to Processing" Prog. Polym. Sci. 1539(28) 2003.

12. Valadares, L. F., Leite, C.A.P., Galembeck, F., "Preparation of natural rubbermontmorillonite nanocomposite in aqueous medium: evidence for polymer-platelet adhesion" Polymer 672(47) 2006.
13. Varghese, S.; Gatos, K.G.; Apostolov, A. A.; Karger-Kocsis, J., "Morphology and Mechanical Properties of Layered Silicate Reinforced Natural and Polyurethane Rubber Blends Produced by Latex Compounding" J. Appl. Polymer Sci. 543 (92) 2004.

14. Wu, J.; Lerner, M. M., "Structural, Thermal and Electrical Characterization of Layered Nanocomposites Derived from Sodiunmontmorillonte and Polyethers" Chem. Mater. 835 (5) 1993.

15. Wu, Y. P.; Wang, Y. Q.; Zhang, H. F.; Wang, Y. Z.; Yu, D. S.; Zhang, L. Q.; Yang, J., "Ruber-preistine clay nanocomposite prepared by co-coagulating latex and clay suspension" Comp. Sci. Technol. 1195 (65) 2005.

Correspondencia: hdccoll@gmail.com

Recepción de originales: junio 2013

Aceptación de originales: diciembre 2013 